\title{
Neonatal programming of piglet gut health and postnatal effects by maternal transfer of phytogenic compounds supplemented in gestating and lactating hyperprolific sows
}

\section{David Reyes-Camacho}

Universitat Autonoma de Barcelona

José Francisco Pérez

Universitat Autonoma de Barcelona

\section{Ester Vinyeta}

Delacon Biotechnik GmbH

\section{Tobias Aumiller}

Delacon Biotechnik GmbH

\section{Lourdes Criado-Mesas}

CRAG

\section{L.M. Palade}

Institutul National de Cercetare-Dezvoltare pentru Biologie si Nutritie Animala

Ionelia Taranu

Institutul National de Cercetare-Dezvoltare pentru Biologie si Nutritie Animala

Josep Maria Folch

Universitat Autonoma de Barcelona

Jan Dirk Van der Klis

Delacon Botechnik GmbH

David Solà-Oriol ( $\nabla$ david.sola@uab.cat)

Universitat Autonoma de Barcelona https://orcid.org/0000-0001-8365-340X

\section{Research}

Keywords: Hyperprolific sows, Maternal transfer, Neonatal programming, Phytogenic compounds, Piglet gut health, Postnatal growth performance

Posted Date: August 5th, 2020

DOI: https://doi.org/10.21203/rs.3.rs-52150/v1 
License: (c) (i) This work is licensed under a Creative Commons Attribution 4.0 International License. Read Full License 


\section{Abstract}

Background The improvement of sow prolificacy by breeding has increased the number of piglets produced per sow per year. However, intrauterine crowding and/or intrauterine growth restriction typically observed in hyperprolific sows impairs foetal growth which has decreased the average individual birthweight, with a larger proportion of low birthweight (LBW) pigs born per litter with poor gut development and compromised postnatal growth performance. Phytogenic compounds (PC) are plantderived natural bioactive substances that can be used in livestock production as feed additives to promote animal health and production efficiency. This research aims to study if a specific blend of PC (BPC) supplemented in gestating and lactating hyperprolific sow diets may promote pre- and postnatal maternal effects on performance and oxidative status of sows and their offspring, colostrum-milk features, and piglet gut health-related gene expression and morphology. Forty DanBred hybrid line Landrace $x$ Yorkhire gilts and sows (parities $0-7$ ) were randomly allocated by parity number and body weight into two dietary treatments including unsupplemented Control $(n=20)$ or Control diets supplemented with $1 \mathrm{~g} / \mathrm{kg}$ feed of BPC $(n=20)$ throughout gestation and lactation.

Results Several dietary PC from the supplemented BPC were transferred to the placental fluid and milk. The BPC supplementation during gestation enhanced the litter size, antioxidant status, and colostrum protein content of sows. Jejunal histomorphology of neonate piglets, and intestinal-function gene expression related to nutrient transport, antioxidant, innate immune response, and digestion were improved in the BPC group. For both, sows and piglets, plasma antioxidant activity of CAT and SOD enzymes were enhanced. For suckling piglets, jejunal expression of genes related to gut barrier function was improved and piglet weight gain from birth to weaning was enhanced in the BPC group.

Conclusions Dietary supplementation of BPC in gestating and lactating diets for hyperprolific sows improves sow's reproductive performance and colostrum composition, with a significant strength of the antioxidant status of sows and their offspring. The prenatal and postnatal maternal transfer (placental fluid and milk) of BPC to the offspring would influence on the neonatal programming and postnatal of piglet's gut health, with advantageous effects on piglet's growth performance.

\section{Background}

Hyperprolific dam-line sows are characterized by the high number of foetuses in utero at late gestation, but relatively poor survival through the peri-parturient period, and lower and less uniform piglets' birth weights [1]. In fact, intrauterine crowding and intrauterine growth retardation are considered the main causes of piglet low birth weight (LBW) in large litters [2]. Foetal growth is a complex biological process influenced by genetic, epigenetic, and maternal environmental factors [3]. These factors influence the uterine capacity, which is limited by placental size and functionality, blood flow, delivery of maternal oxygen and nutrient to the foetus, as well as the balance between hormonal and metabolic pathways, that in turn determine litter size and birth weights in swine [4]. 
Modifications in foetal nutrition and endocrine status may regulate physiological and metabolic changes, and, ultimately, influence offspring phenotype and postnatal growth rate [5]. Amniotic fluid is recognized mainly for its growth-promoting properties on the foetal intestine [6]. An imbalanced allocation of limited nutrients among foetuses during gestation, mediated by placental dysfunction, may also affect the intestinal development and microbial colonization of the LBW piglet [7]. Indeed, intestinal dysbiosis is one of the major causes of morbidity and impaired growth performance in LBW piglets, since developmental pattern of intestinal barrier, as well as, transcriptomic and microbiota profiles, are highly affected in the intestine of the neonate pigs, resulting in a higher propensity to develop certain health disorders, including metabolic and digestive disorders and problems linked to the immune system [8], [9].

In term of foetal programming, maternal nutrition status during foetal development may result in a permanent imprint by changing the epigenetic state of the foetal genome and their gene expression [10]. It is reported that several factors including inherent genetic programming, microbial colonization, and colostrum and milk components are involved in the neonatal epithelial barrier establishment [11]. Mammary secretions (colostrum-milk) contain similar bioactive compounds as amniotic fluid which act as biological signals with high similitude, modulating the intestinal environment and immature immune status in new-born piglets and later in life [12], [13]. Recent studies have shown that maternal transfer of several phytogenic substances supplemented to the sow diet into amniotic fluid, colostrum and milk may influence reproductive sow performance, colostrum-milk features [14] and piglet's growth performance [15].

Phytogenic compounds (PC) is a term used to describe plant-derived natural bioactive compounds, including for example terpenes, polyphenols, and glycosides [16]. Although their modes of action are not fully understood, the literature describes a series of effects of PC suitable for promoting livestock health and production efficiency, including digestive and immune stimulation, anti-inflammatory and antimicrobial activity, intestinal microbiota modulation, and antioxidant effects [17], [18], as well as oestrogenic and hyperprolactinaemic properties [19] and influence on placental functionality [20].

For the present study, it was hypothesised that a blend of PC (BPC) supplemented to the feed of gestating and lactating sows can be transferred both pre- and postnatally to piglets, showing an influence on performance and oxidative status of sows and piglets. Therefore, BPC were supplemented into the diet of hyperprolific sows during gestation and lactation to study the effects on reproductive performance, colostrum-milk features, and antioxidant status of sows and their offspring, as well as the effect on piglet gut-health (gene expression and morphology) and growth performance.

\section{Methods}

\section{Experimental design, animals, and housing}

The experiment was arranged as a randomised complete-block design under commercial farm conditions in which 40 DanBred hybrid line (Landrace x Yorkshire) gilts and sows (parity 0 to 7) were randomly distributed by parity number and body weight (BW) into 2 dietary treatment groups. As individual 
controlling inseminated and gestating sows, the sow was considered as experimental unit at service and during gestation period ( $n=20$ per treatment). During lactation, the sow with her litter was considered as experimental unit ( $n=14$ per treatment). Difference between the initial number of sows (breeding) and sows allotted at farrowing (lactation) corresponds to sows that showed physiological issues (heat failure [gilts], repeated oestrous, or abortion). After breeding, sows were fed unsupplemented control diets during gestation and lactation (Control) or the control diets supplemented with $1 \mathrm{~kg} / \mathrm{MT}$ of phytogenic compounds (Delacon Biotechnik GmbH., Engerwitzdorf, Austria) during the whole gestation and lactation period (BPC). From mating ( $0 \mathrm{~d}$ ) until confirmed gestation ( $35 \mathrm{~d}$ ), sows were assigned to individual cages $(1.8 \times 0.8 \mathrm{~m})$. On day 35 , sows were allotted by parity and BW into group pens of 10 sows/pen $(5.0 \times 5.0$ $\mathrm{m})$ until day 110 of gestation. Thereafter, sows were moved to the farrowing unit, where they were placed in individual farrowing pens $(2.0 \times 2.6 \mathrm{~m})$ mounted over a partially slatted floor with a heated floor pad for piglets and ad libitum access to water for both sows and piglets until weaning. The environmental temperature in the farrowing room was automatically controlled $\left(22^{\circ} \mathrm{C}\right)$. Within $24-48 \mathrm{~h}$ post-farrowing, litter size was standardised to 15 piglets/litter by cross-fostering (within the respective treatment group). Experimental diets were provided to sows as pellets during gestation and lactation.

\section{Diets and feeding system}

The experimental diets offered in the gestation and lactation period were formulated to meet or exceed nutrient requirements for DanBred sows [21], with adaptations based on Spanish recommendations for gestating and lactating sows [22] (Table 1). Sow experimental diets were the control diets plus $1 \mathrm{~g} / \mathrm{kg}$ of BPC supplement containing limonene, anethole, borneol, p-cymene, and eucalyptol, added as essential oils. Sows were fed their corresponding treatment diet as follows: $2.1 \mathrm{~kg} / \mathrm{d}$ from weaning to service, and a mean of $2.9 \mathrm{~kg} / \mathrm{d}$ from service to $\mathrm{d} 35$ of gestation, based on individual body condition. Thereafter, sows were fed $2.5 \mathrm{~kg} / \mathrm{d}$ from $35 \mathrm{~d}$ to $110 \mathrm{~d}$ of gestation of the gestation diet (flat line). Each gestating pen was equipped with enough mechanical free access self-closing semi-cage without pneumatic actuators (Rotecna, Lleida, Spain) to keep animals individually monitored during feeding. From $110 \mathrm{~d}$ of gestation and during lactation, sows were fed their corresponding treatment diet for ad libitum intake.

\section{Sampling}

Sow blood samples ( $6 \mathrm{~mL} / \mathrm{sow}$ ) were collected by caudal venepuncture on days 0 (service), 35 (confirmed gestation), 110 (late gestation) ( $n=20$ per treatment), and day 2 post-weaning ( $n=12$ per treatment). From the same subset 8 out of 12 sows per treatment were chosen by parity to select one piglet with average BW from their litter for collection of blood samples $(6 \mathrm{~mL} /$ piglet) by jugular vein puncture before being euthanized at farrowing (without sucking colostrum), and day 20 of lactation ( $n=$ 8 per treatment). After blood sampling, plasma/serum samples were obtained by centrifugation at 2,000 $\mathrm{xg}$ for $15 \mathrm{~min}$ and stored at $-20^{\circ} \mathrm{C}$ until analysis.

The placental fluid samples ( $60 \mathrm{~mL} / \mathrm{sow}$ ) were collected by extrusion at farrowing from the same subset of sows ( $n=12$ per treatment). Colostrum and mature milk samples ( $30 \mathrm{~mL} / \mathrm{sow}$ ) were collected within $12 \mathrm{~h}$ of farrowing ( $n=12$ per treatment), and at day 20 of lactation ( $n=12$ per treatment) from all 
functional mammary glands by hand milking after injection oxytocin (dose $2 \mathrm{~mL}$ ). Colostrum-milk samples were not filtered and immediately put on ice followed by storage at $-20^{\circ} \mathrm{C}$ until analysis.

Jejunum tissue samples were immediately collected after euthanasia from the same subset of sampled piglets ( $n=8$ per treatment). Before being euthanized, pigs were anesthetized by intramuscular injection of a 1-mL final mixture contained $100 \mathrm{mg}$ telazol, $50 \mathrm{mg}$ ketamine, and $50 \mathrm{mg}$ xylazine (2:1:1) in $1 \mathrm{~mL} / 23$ $\mathrm{kg}$ of BW. After anaesthesia, pigs were euthanized by jugular vein injection using sodium pentobarbital $0.5 \mathrm{~mL} / \mathrm{kg}$ of BW. Obtained tissue for gene expression analysis (approx. $1 \mathrm{~cm}^{2}$ ) was placed in $1 \mathrm{~mL}$ of RNAlater (Applied Biosystems, Foster City, CA, United States) and stored at room temperature $\left(25^{\circ} \mathrm{C}\right)$ for $24 \mathrm{~h}$ before storage at $-80^{\circ} \mathrm{C}$ until analysis. Additional jejunum tissue samples (approx. $2 \mathrm{~cm}^{2}$ ) were placed in individual tubes containing formaldehyde solution for histologic determination.

\section{Data recording and laboratory analyses}

Productive performance parameters of sows including BW and back fat thickness were measured at $\mathrm{d} 0$, d 35 and d 110 of gestation, and at weaning. The back fat thickness was measured by digital Bultrasound (model WED-3000V, Welld®, Shenzhen, China) at P2 position (left side of the midline at last rib and $7.5 \mathrm{~cm}$ to the spine). In addition, the individual average daily feed intake (ADFI) of sows during gestation was controlled by weighing the feed offered and checking for refusals [mechanical free access self-closing semi-cage (Rotecna ${ }^{\circledR}$, Lleida, Spain)]. During lactation, ADFI of sows was recorded (twice a day) by weighing feed offered and refused using a feeding ball system [ad-libitum pan with ball mechanism for farrowing (Rotecna ${ }^{\circledR}$, Lleida, Spain)]. Sows reproductive performance (litter size) at farrowing, considered the total number of live, dead, or mummified piglets. Whereas litter performance included the number and individual BW of piglets at birth, cross-fostering, d 20 of lactation, and weaning. In addition, the number of days from weaning to oestrus (weaning to oestrus interval) was recorded.

Maternal transfer of BPC through the placental fluid and milk were determined by solid-phase microextraction, gas chromatography-mass spectrometry (Servei d'Anàlisi Química, Autonomous University of Barcelona UAB, Spain) according the volatile PC profile characterised in the tested supplement. Results of the relative peak abundance were estimated based on the ratio (abundance/retention time) for each volatile compound and expressed as percentage of proportional increased concentrations in relation to assigned reference values (basal value $=100$ ) in control sows.

The oxidative status of sows and piglets was determined by measuring the antioxidant enzyme activities of catalase (CAT), and superoxide dismutase (SOD) in plasma/serum using the standardised Cayman Chemical Kits (both Cayman Chemical, Ann Arbor, USA), according the manufacturer's protocol, applied on a Tecan microplate reader (Tecan, SunRise, Austria). The CAT activity was assayed at $540 \mathrm{~nm}$ and results were expressed as nanomoles per minute per millilitre $(\mathrm{nmol} / \mathrm{mL})$. Activity of SOD was measured using the cytochrome $c$ and xanthine oxidase coupled assay at $440-460 \mathrm{~nm}$ and results expressed as units per millilitre $(\mathrm{U} / \mathrm{mL})$. Plasma lipid peroxidation was measured based on the thiobarbituric acidreactive substances (TBARS) concentrations as follows: plasma/serum was mixed with deionized water, 
$0.5 \mathrm{~N} \mathrm{HCL}$, and thiobarbituric acid and incubated at $95^{\circ} \mathrm{C}$ for $15 \mathrm{~min}$. Thereafter, using a Tecan Infinite M200 microplate reader, measurement was made in fluorescence mode (maximum excitation $515 \mathrm{~nm}$ : emission range $548 \mathrm{~nm}$ ), and results were expressed as nanomoles of malondialdehyde per millilitre $(\mathrm{nmol} / \mathrm{mL})$ of plasma using 1,1,3,3-tetramethoxypropane as standard.

Colostrum and milk composition were determined by standardised methods as follows: crude protein by the Kjeldahl method AOAC 991.22. Crude fat by the Röse-Gottlieb method AOAC 905.02; and lactose by the Luff-Schoorl volumetry method B.O.E. num. 52 R.D. 2257/ 1994.

Gene expression analysis was performed using quantitative real-time PCR (RT-qPCR) as follows: from $100 \mathrm{mg}$ of jejunum tissue was extracted the total RNA using the RiboPure Kit (Ambion, Life technologies, Carlsbad, CA), by following the manufacturer's protocol. Quantification of the total RNA was measured using a Nano-Drop ND-1000 spectrophotometer (NanoDrop products, Saveen Werner, Malmö, Sweden), and RNA integrity was checked using an Agilent Bioanalyzer-2100 equipment (Agilent Technologies). Around $1 \mu \mathrm{g}$ of total RNA in a final volume of $20 \mu \mathrm{L}$ was used for cDNA synthesis with random primers using the High-Capacity cDNA Reverse Transcription Kit (Applied Biosystems, Foster City, CA, United States). A total of $25 \mathrm{ng}$ of cDNA sample was pre-amplified, using a TaqMan PreAmp Master Mix (Life Technologies, Foster City, CA) and a Pooled TaqMan Gene Expression custom assay following the manufacturer's protocol. The pre-amplified product was diluted 1:10 with 0.1X Tris-EDTA pH 8.0 and analysed per duplicate in Taqman Open Array gene expression plates custom-designed in a QuantStudio 12K Flex Real-Time PCR system (ThermoFisher Scientific, Waltham, MA, USA). Inter-plate controls were used to check the replication of results from different plates.

A list of 56 genes related to gut-health function was previously selected based on the bibliography, and included 4 reference genes (see Supplementary Table 1). Primers were designed by spanning exon-exon boundaries using PrimerExpress 2.0 software (Applied Biosystems), and genomic DNA amplification and primer dimer formation were controlled. Gene expression data was collected and analysed using the ThermoFisher Cloud software 1.0 (Applied Biosystems), and the $2^{-\Delta \Delta C t}$ method for relative quantification (RQ) was applied, using the sample with the lowest expression as a calibrator.

For the histomorphology evaluation jejunum samples were fixed for $24-48 \mathrm{~h}$ in neutral-buffered $10 \%$ formalin. After dehydration and embedding in paraffin wax, sections of approx. $\sim 3 \mu \mathrm{m}$ were stained with haematoxylin and eosin. Villus height (VH), crypt depth (CD), number of intraepithelial lymphocyte (IEL), and number of goblet cells (GC) were measured blinded in 10 well-oriented villi and crypts by the same person using a light microscope (BHS, Olympus, Barcelona, Spain).

\section{Statistical analyses}

Statistical analyses were performed by different procedures of the statistical package SAS 9.4 (SAS Inst. Inc., Cary, NC). The sow was considered as experimental unit at service and gestation period, while pen was included as random effect. During lactation, the sow with her litter was considered as experimental unit. Corresponding data to performance, maternal transfer of BPC, antioxidant status, colostrum and 
milk composition, as well as intestinal gene expression and morphology were fit to a general linear model (two-way) by using the GLIMMIX procedure:

$\mathrm{Y}_{\mathrm{ij}}=\mu+$ treat $_{\mathrm{i}}+$ parity $_{\mathrm{j}}+\varepsilon_{\mathrm{ij}}$

where $Y_{i j}$ relates to each observation of the outcome variable, $\mu$ is the global mean, treat $t_{i}$ is the main effect of treatment, parity ${ }_{\mathrm{j}}$ is the covariate effect for sow parity number and, $\varepsilon_{\mathrm{ij}}$ is the experimental error term. Therefore, all data were analysed considering the treatment as main effect, and results are presented as least square (LS) means with their corresponding SEM. All data were checked for outliers before the statistical analysis $t$ with outliers defined by a deviation of $\geq 2.0$ times the SEM. Normality and equal variances were verified in all continuous variables using the Shapiro-Wilk test by using the UNIVARIATE procedure. Tukey adjust test was considered for all multiple comparisons between treatments. Finally, effects were considered significant when the probability value $P$ was $\leq 0.05$ and considered as 'trends' with $0.05<P \leq 0.10$.

\section{Results}

\section{Performance}

Regarding the performance of sows (Table 2), no differences were observed between treatments on the BW, back fat thickness, ADFI during gestation, and weaning to oestrus interval $(P>0.05)$. However, compared to control sows, the standardized lactation ADFI (d 20), and the standardized total feed intake (gestation plus standardized lactation $\mathrm{FI})$ tended to be increased $(P=0.085)$, and $(P=0.071)$ respectively, in BPC group. Concerning the sows reproductive and piglet performances (Table 3), BPC supplementation during gestation tended to increase the total number of piglets born $(P=0.084)$, and the number of piglets born alive $(P=0.077)$, while tended to decrease $(P=0.092)$ the born alive piglet weight. No differences were observed between treatments on the sow litter size and piglet weight at cross-fostering, lactation d 20, and weaning, as well as, on the lactation length $(P>0.05)$. Nonetheless, the individual piglet BW gain from birth to weaning was higher $(P=0.018)$ in the BPC group.

\section{Prenatal and postnatal maternal transfer of BPC}

Results on BPC transfer from feed into placental fluid (prenatal) and milk (postnatal) are given in Fig. 1. Analysis of placental fluid showed an increase $(P<0.05)$ in relative concentrations of limonene, anethole, borneol, p-cymene, and eucalyptol in the BPC group when compared with the Control. During lactation, the relative concentration of limonene, anethole, and p-cymene were increased $(P<0.05)$ in milk of sows from the BPC when compared to the Control, while no differences were observed for borneol $(P>0.05)$, and eucalyptol was not detected in milk (considering a limit of detection of $16 \mathrm{ppb}$ ).

\section{Colostrum and milk composition}


The effects of dietary BPC supplementation on colostrum and milk composition are described in Fig. 2. Results indicated that BPC supplemented during gestation increased $(P<0.001)$ the colostrum protein content without affecting $(P>0.05)$ colostrum fat and lactose contents. There were no differences $(P>$ $0.05)$ on milk nutrient composition.

\section{Sows and piglet's antioxidant status}

The physiological state and dietary treatments influence on plasma antioxidant status of the sows and their offspring is given in Fig. 3. Briefly, results showed no differences between treatments on basal values at $\mathrm{d} 0$ and at day 110 of gestation for CAT, SOD, and TBARS in sows. Comparing the BPC group to the control at early gestation (d 35), CAT activity and TBARS levels were increased $(P<0.05)$, whereas SOD was decreased $(P<0.05)$. For day 2 post-weaning SOD and CAT activities in sow plasma were increased $(P<0.05)$ in the BPC group while no difference was observed for TBARS $(P>0.05)$.

The BPC group showed increased TBARS-values in plasma of newborn piglets. At lactation $\mathrm{d} 20$ both the CAT and SOD activities were higher $(P<0.05)$ in BPC group while no difference was observed for TBARS.

\section{Piglet's jejunum gene expression}

Results for the jejunum gene expression analysis are shown in Fig. 4. Although all genes were influenced by treatments, only the following showed significant differences: In neonate piglets samples an upregulation $(P<0.05)$ was observed for solute carrier family 1 member 2 (SLC11A2), monocarboxylate transporter 1 (SLC16A1), and solute carrier family 39 member 4 (SLC39A4), superoxide dismutase (SOD), and intestinal alkaline phosphatase (ALPI), while interferon gamma (INF- $\gamma$ ) was downregulated $(P<0.05)$ in the BPC group when compared to the Control. For suckling piglets $(\mathrm{d} 20)$ an upregulation $(P<0.05)$ of barrier function genes mucin 2 (MUC2), claudin-4 (CLDN4), claudin-15 (CLDN15), and trefoil factor 3 (TFF3) was observed when comparing the BPC group to the Control.

\section{Piglet's jejunum histomorphology}

Jejunum histomorphometry measurements of new-born piglets are summarized in Table 4. Results indicated that $\mathrm{BPC}$ increased the $\mathrm{VH}(P=0.013), \mathrm{VH}: \mathrm{CD}$ ratio $(P=0.024)$, and $\mathrm{GC}$ density $(P=0.033)$ in neonate piglets. However, no significant differences $(P>0.05)$ were observed on $\mathrm{CD}$, and IEL density. No differences $(P>0.05)$ between treatments on jejunal morphology were observed in suckling piglets (data not shown).

\section{Discussion}

The results of the study are discussed separately regarding the prenatal and postnatal effects.

\section{Prenatal effects of BPC on sows and their offspring}


Hyperprolific dam-line sows have high quantity of foetuses in utero with increasing nutritional requirements, especially during the last third term of gestation. To support the requirements of the growing foetus, placental size and exchange surface area requires to be increased. Such adaptations contribute to optimize nutrient availability during late gestation and improve foetal growth and survival [23]. Thus, conditions regarding placental vascularization are critical for the intrauterine function, and deficiencies may result in reduced BW of piglets born alive and poor survival especially during the periparturient period[2]. In this context, two biological processes are related with tissue neovascularization. While vasculogenesis occurs during embryo development, resulting in the de novo formation of blood vessels by the accumulation of angioblasts (endothelial precursors), angiogenesis is involved in tissue growth and regeneration, by extension of vasculature formed from vasculogenesis [24].

According to Rajasekar et al. [20] several dietary natural compounds (e.g. polyphenols, terpenoids and plant extracts) have been associated to potential modes of action of modulating angiogenesis pathways. In general, they may modulate angiogenesis by down-regulating the secretion of angiogenesis factors such as VEGF, FGF, Ang, MMPs, or by inhibiting receptor mediated signalling pathways (PI3K/Akt, ERK) involved in hypoxic and inflammatory conditions. An effect of BPC on placental neovascularization might be associated to alterations (imbalance) between pro- and anti-angiogenic factors [20].

Recent studies have shown the transfer of PC such as anethole, cinnamaldehyde, eugenol [25], limonene and carvone [15] from the feed to amniotic fluid of sows during gestation, with effects on reproductive performance and mammary secretions [14], as well as, the antioxidant status in gestating sows [26]. The results of the present study are in line with such findings with the relative increase of BPC limonene, anethole, borneol, p-cymene, and eucalyptol in the sow's amniotic fluid compared to the Control group. It was previously reported that prenatal exposure of foetus to specific BPC containing eucalyptol, p-cymene, linalool, anethole, and thymol essential oils along the gestation period increased the litter size (born alive) [14]. This may reflect improvements on reproductive parameters as the ovulation rate, embryonic survival, and/or uterine and placenta function [1].

The modes of action on tendencies observed in this study regarding the reproductive performance suggest an influence of the supplemented BPC on placental functionality, however, remains unclear. Possibly the observed effect on the sows' oxidative status is related to this effect. A potential of improving the antioxidant status of sows with BPC was shown already by Meng et al. [27] where dietary resveratrol (polyphenol) supplementation during gestation, increased both the CAT and GSH-Px plasma levels by modulation of the Keap1-Nrf2 pathway and Sirt1 in the placenta. In the present study, it was shown that BPC sows had higher plasma TBARS levels and CAT activity, but lower SOD levels during the early period of gestation ( $d 35$ ). The changes could indicate an antioxidant response (acquisition of tolerance) to protect cell from endogenous free radical attacks [28].

Upon birth, a critical factor for further development of the new-born piglets is their colostrum intake. Quesnel and Farmer [29] describe that sow nutrition during late gestation may influence colostrogenesis, which starts approximately 10 days before farrowing, englobing both, the synthesis of milk-specific 
constituents and the transfer of IgG into lacteal secretions. During colostrogenesis, tight junctions between mammary epithelial cells are leaky, thereby allowing paracellular transfer of constituents from maternal plasma to the alveolar compartment, especially hormones, growth factors and Ig. It is noteworthy that colostrum protein content in the BPC group was increased. Therefore, it would be of high interest for further studies to investigate the proportion of different compounds within the protein fraction of the colostrum in BPC supplemented sows. An increased amount of growth factors and immunoglobulins could offer an advantage for piglets in their early-life development, especially until their own immune system is fully functional.

Dietary phytochemicals have been shown to offer a potential for modulating a range of biological process and pathways (e.g. nutrient transport, immune and inflammatory response, and muscle physiology and metabolism) with transcriptome profiles in human adults [30], pigs [31], and poultry [32]. The gene expression analysis performed in the present study on the jejunum of new-born piglets showed that supplementation of BPC to gestating sows up-regulated mRNA levels of SLC11A2, SLC16A1, $S L C 39 A 4$, and $A L P I$ genes, which are related to gut function in neonate piglets. Specifically, the solute carrier family gene $S L C 11 A 2$ is involved in intestinal uptake of divalent metals, especially $\mathrm{Fe}^{2+}$ [33], SLC16A1 plays a role in the luminal absorption of short-chain fatty acids [34], SLC39A4 is required for intestinal uptake of zinc [35], while $A L P /$ is enterocyte differentiation dependent involved in phosphate digestion and fat absorption, as well as detoxification of pathogenic bacterial [36]. Upregulation of these genes suggests a potential improvement on the capacity for intestinal nutrient uptake and transport. Likewise, the observed upregulation of the $S O D$ gene suggests an effect on the antioxidant system [37]. The IFN- $y$ gene was downregulation in the BPC compared to the Control group, and this may be positively related to the innate immune response [34], as auto-regulatory loop of local inflammation [38].

To our knowledge, there are no studies in swine reporting on effects of the prenatal maternal transfer of PC on the neonatal programming regarding parameters for the intestinal integrity. The term 'gut health' encompasses a number of physiological and functional mechanisms including morphological, anatomical, microbial, enzymatic and immunological, and the interactions between these components [39]. In particular, atrophied villus structure and hyperplasia indicated by a decreased VH and increased CD [40], as well as, dysfunctionality of specialized epithelial cell types such as goblet cells [11] are compatible with an impaired of the mucosa (epithelium) GIT. It was demonstrated that IUGR conditions affected weight and structure (villous size) of the intestine and enhanced counts of adherent bacteria to the epithelium [8]. In this study, new-born piglets in the BPC supplemented group showed higher VH, ratio of $\mathrm{VH}: \mathrm{CD}$, and goblet cell density when compared with new-born piglets from the Control group, indicating that BPC would act in opposition to IUGR intestinal impairs.

Taking together the observed response on gene expression, intestinal histomorphology, nutrient transporters, oxidative status, and immune parameters by supplementation of BPC group suggest prenatal programming of some biological mechanisms involved in the development of the foetus until birth. Considering the direction of differences, although intrauterine space is not modified resulting in 
lower and less uniform new-born piglet BW, BPC supplementation to the sow's gestation diet could have increased supply of total nutrients, resulting in improved perinatal survival.

\section{Postnatal effects of BPC supplementation on sows and piglets}

The analysis of volatile compounds in milk at day 20 of lactation showed differences in the transfer of specific BPC. Relative concentrations of limonene, anethole, and p-cymene were increased in milk of BPC supplemented sows. However, no evidence for increased milk concentrations of eucalyptol and borneol were found in the BPC group in contrast to findings from placental liquid. Potentially these differences could be related to the bioavailability and chemical structure of the analysed BPC (e.g., molecular weight, size, lipophilicity, hydrophilicity, and charge) [41]. This has an especial interest because Kirsch and Buettner [42] reported a carry-over of eucalyptol into human milk. Song et al. [43] mentioned that differences in the relative concentrations of phytochemicals such as flavonoids and carotenoids in human milk may be a result of several factors, including dietary exposure, stability in the milk matrix, efficiency of absorption/metabolism, and transfer from plasma to milk.

Regarding possible effects of the BPC present in the maternal diet, it was previously reported that they can have an influence on nutritional programming and/or sensory conditioning and further performance during the perinatal period. For example, a study by Val-Laillet et al. [13] used two different blends of PC (limonene and cinnamaldehyde, or menthol, carvone, and anethole) as sensory feed additives in gestating and lactating sows. These supplemented PC were detected in both colostrum and milk of the sows and suckling pigs that were exposed to feed additives via the maternal diet showed improved ADG and ADFI compared to piglets from sows without supplementation of PC. This type of perinatal exposure to $\mathrm{PC}$ could influence nutritional programming and/or sensory conditioning during an important time for the development of flavour preferences, appetite regulation, and nutritional programming, both in humans and pigs [44].

In the present study, lactating sows receiving the BPC tended to have increased ADFI and their offspring showed higher BWG from birth to weaning than piglets from sows receiving the Control. Similar to these findings, Tan et al. [45] described a tendency to increase the feed intake of lactating sows after dietary supplementation of $15 \mathrm{mg} / \mathrm{kg}$ oregano EO, with improved piglets ADG from birth to day 21 of lactation. In addition, Wang et al. [46] reported an improved ADFI and milk yield during lactation and in addition the body weight of piglets at weaning was increased with dietary supplementation of $0.5 \%$ of star anise during gestation and lactation of sows. They indicated that star anise in diet improved the lactation performance of sows by increasing concentrations of insulin-like growth factor- 1 in milk and prolactin in serum of sows.

These effects may be linked not only to feed intake of the animals, but also their oxidative status. As catabolic conditions during late gestation and lactation increase the production of reactive oxygen species it is well accepted that hyperprolific sows may be under systemic oxidative stress [47], [45]. This can affect not only fertility and well-being of sows, but also their piglets' [48], as oxidative factors can be transferred to the offspring through mammary secretions [49]. Therefore, dietary programs reducing 
oxidative stress in sows are of interest. A potential of PC in this regard was shown for example by the previously cited study of Tan et al. [45] where supplementation of $15 \mathrm{mg} / \mathrm{kg}$ oregano EO during gestating and lactating resulted in reduced TBARS serum levels in sows on early lactation when compared with early gestation levels. In addition, evidence has shown that dietary supplementation with certain botanical functional substances; alleviate oxidative stress in sows and their offspring. For example, Meng et al. [27] described a positive effect of dietary supplementation of resveratrol, a plant phenol, during lactation, which affords protection against inflammation and oxidative stress, improving the SOD and CAT levels in both plasma and milk of sows and piglets. A similar result was obtained in the present study by the BPC supplementation during lactation, with an increase in both CAT and SOD plasma levels of the lactating sows and their piglets.

Especially targeting the endogenous antioxidant response of animals might be promising target to increase resilience against oxidative stress. Particularly SOD and CAT are interesting enzymes in this regard, as SOD acts as a primary defence against superoxide anion radical and converting it to $\mathrm{H}_{2} \mathrm{O}_{2}$, while CAT, a haem-containing enzyme, in turn detoxifies $\mathrm{H}_{2} \mathrm{O}_{2}$ to water and non-ROS, protecting the tissues from high peroxide levels [50]. Studies in rodents demonstrated the potential of oral treatment with D-limonene to increase the activities of both SOD and CAT, reducing the oxidative stress in diabetic rats by decreasing lipid peroxidation [51]. Similar results were obtained by De Oliveira et al. [52] after dietary supplementation of $p$-cymene in mice. The level of lipid peroxidation and nitrite content was reduced while CAT and SOD activities increased. These findings suggest that the antioxidant effect of these BPC (containing limonene and p-cymene) may be due to positive modulation of the activity of these antioxidant enzymes.

the overall metabolism, physiology, disease status and performance of young piglets,an optimally functioning gastrointestinal tract the gene expression analysis of jejunum samples from suckling piglets showed that BPC supplementation in lactating sows resulted in an upregulation of some genes related to gut barrier function (MUC, CLDN4, CLDN15, TFF3) which could act as possible markers for gut barrier functionBriefly, MUC is related to intestinal secretion of gel-forming mucin [54], CLDN4 and CLDN15 involved in the transmembrane protein sealing functions, and mucosal differentiation, respectively [55], as well as, TFF3 functioning as mucosal protection and epithelial restitution [54]. In further studies it would be interesting to study if these effects on intestinal barrier genes together with the observed histomorphological changes in the jejunum of piglets from the BPC fed sows are related to the observed improvement of BW gain from birth to weaning.

\section{Conclusions}

The present study demonstrated that dietary BPC supplemented to hyperprolific sows during gestation and lactation influenced the sow's reproductive performance, colostrum composition, and antioxidant status of the sows and their offspring. Open questions remain regarding the mode of action of the BPC on observations such as foetus survivability and development programming of gut functions and morphology in neonates, which need to be clarified in future studies. However, the combined prenatal and 
postnatal effects of the BPC supplementation observed in this study suggest a benefit for piglet's that resulted in improved growth performance from birth to weaning.

\section{Abbreviations}

ADFI: Average daily feed intake; ALPI:Intestinal alkaline phosphatase; Ang:Angiopoietins; ANPEP:Aminopeptidase-N; BPC:Blend of phytogenic compounds; BW:Body weight; cDNA:complementary deoxyribonucleic acid; CAT:Catalase; CD:Crypt depth; CLDN4:Claudin-4; CLDN15:Claudin-15; EO:Essential oil; ERK:Extracellular signal-regulated kinases; FEDNA:Spanish Federation for the Development of Animal Nutrition; FGF:Fibroblast growth factor; FI:Feed intake; GC:Goblet cells; GIT:Gastrointestinal tract; GSHPx:Glutathione peroxidase; IgG:Immunoglobulin G; IDO:Indoleamine 2, 3- dioxygenase; IEL:Intraepithelial lymphocyte; IL-10:Interleukin 10; INF-y:Interferon gamma; IUGR:Intrauterine growth restriction; LBW:Low birth weight; LS:Least square; MMP:Matrix metalloproteinase; VEGF stands to be a mitogen for MUC2:Mucin 2; NO:Nitric oxide; PC:Phytogenic compounds; PCR:Polymerase chain reaction; PPARGC1a:Peroxisome proliferative activated receptor gamma coactivator 1 alpha; PI3K/Akt:Phosphatidylinositol 3'- kinase; RNA:Ribonucleic acid; ROS:Reactive oxygen species; RT-qPCR:Reverse-transcriptase real-time polymerase chain reaction; SD:Standard deviation; SEM:Standard error of the mean; SLC11A2:Solute carrier family 1 member 2; SLC16A1:Solute carrier family 16 Member 1; SLC39A4:Solute carrier family 39 member 4; SOD:Superoxide dismutase; TBARS:Thiobarbituric acid-reactive substances; TFF3:Trefoil factor 3; VEGF:Vascular endothelial growth factor; VH:Villus height.

\section{Declarations}

\section{Ethics approval and consent to participate}

All animal procedures were reviewed and approved by the Animal Ethics Committee of the Autonomous University of Barcelona, according to the directive European Parliament, 2010/63/EU on the protection of animals used for scientific purposes.

\section{Consent for publication}

All authors provide their consent to this publication.

\section{Availability of data and materials}

The datasets used and/or analysed during this study are available from the corresponding author on reasonable request.

\section{Competing interests}


The authors declare that they have no competing interests.

\section{Funding}

This research was funded by Delacon Biotechnik GmbH, 4209 Engerwitzdorf, Austria.

\section{Authors' contributions}

The contributions of the authors were as follows: DRC conducted the animal trial and performed laboratory work; the statistical analysed on all data, and drafted the manuscript. JFP and DSO were the principle investigator, and contributed in conceptualization and experimental design. EV and JDK conducted conceptualization and experimental design. TA supervised the interpretation of the study results, and revised the manuscript. LCM and JMF contributed in the design and set-up of the gene expression studies. LMP determined the antioxidative status in plasma/serum. IT and LMP analysed the results of plasma antioxidative status.

\section{Acknowledgements}

Authors would like to thank the support received from the staff of the Servei d'Anàlisi Química of Universitat Autònoma de Barcelona for the laboratory analysis.

\section{References}

1. Foxcroft GR, Dixon WT, Dyck MK, Novak S, Harding JC, Almeida FC. Prenatal programming of postnatal development in the pig. Soc Reprod Fertil Suppl. 2009;66:213-31.

2. Matheson SM, Walling GA, Edwards SA. Genetic selection against intrauterine growth retardation in piglets: A problem at the piglet level with a solution at the sow level. Genet Sel Evol. 2018;50.

3. Bagnell CA, Bartol FF. Review. Maternal programming of development in the pig and the lactocrine hypothesis. Animal. 2019;2978-85.

4. Vallet JL, McNeel AK, Miles JR, Freking BA. Placental accommodations for transport and metabolism during intra-uterine crowding in pigs. J Anim Sci Biotechnol. 2014;5:1-14.

5. Rekiel A, Wi凶cek J, Batorska M, Kulisiewicz J. Effect of sow prolificacy and nutrition on preand postnatal growth of progeny - A review. Ann. Anim. Sci. 2014. p. 3-15.

6. Siggers J, Østergaard MV, Siggers RH, Skovgaard K, Mølbak L, Thymann T, et al. Postnatal amniotic fluid intake reduces gut inflammatory responses and necrotizing enterocolitis in preterm neonates. Am J Physiol - Gastrointest Liver Physiol. 2013;304:864-75.

7. Jiang L, Feng C, Tao S, Li N, Zuo B, Han D, et al. Maternal imprinting of the neonatal microbiota colonization in intrauterine growth restricted piglets: A review. J Anim Sci Biotechnol. 2019;10:1-8. 
8. D'Inca R, Kloareg M, Gras-Le Guen C, Le Huërou-Luron I. Intrauterine Growth Restriction Modifies the Developmental Pattern of Intestinal Structure, Transcriptomic Profile, and Bacterial Colonization in Neonatal Pigs. J Nutr. 2010;140:925-31.

9. Gaukroger CH, Stewart CJ, Edwards SA, Walshaw J, Adams IP, Kyriazakis I. Changes in Faecal Microbiota Profiles Associated With Performance and Birthweight of Piglets. Front Microbiol. 2020;11:1-14.

10. Wu G, Bazer F, Cudd T, Meininger C. Recent Advances in Nutritional Sciences-Maternal Nutrition and Fetal Development. Nutr. 2004;2169-72.

11. Moeser AJ, Pohl CS, Rajput M. Weaning stress and gastrointestinal barrier development: Implications for lifelong gut health in pigs. Anim Nutr. 2017;3:313-21.

12. Møller HK, Fink LN, Sangild PT, Frøkiær H. Colostrum and amniotic fluid from different species exhibit similar immunomodulating effects in bacterium-stimulated dendritic cells. J Interf Cytokine Res. 2011;31:813-23.

13. Li L, Lv Y, Guan W, Min T, Zhang Y, Chen F, et al. Recent progress of porcine milk components and mammary gland function. J Anim Sci Biotechnol. 2018;9.

14. Reyes-Camacho D, Vinyeta E, Pérez JF, Aumiller T, Criado L, Palade LM, et al. Phytogenic actives supplemented in hyperprolific sows: Effects on maternal transfer of phytogenic compounds, colostrum and milk features, performance and antioxidant status of sows and their offspring, and piglet intestinal gene expression. J Anim Sci. 2020;98.

15. Val-Laillet D, Elmore JS, Baines D, Naylor P, Naylor R. Long-term exposure to sensory feed additives during the gestational and postnatal periods affects sows' colostrum and milk sensory profiles, piglets' growth, and feed intake1. J Anim Sci. 2018;96:3233-48.

16. Stevanović ZD, Bošnjak-Neumüller J, Pajić-Lijaković I, Raj J, Vasiljević M. Essential oils as feed additives-Future perspectives. Molecules. 2018.

17. Lillehoj H, Liu Y, Calsamiglia S, Fernandez-Miyakawa ME, Chi F, Cravens RL, et al. Phytochemicals as antibiotic alternatives to promote growth and enhance host health. Vet Res. 2018;49:1-18.

18. Zeng Z, Zhang S, Wang H, Piao X. Essential oil and aromatic plants as feed additives in nonruminant nutrition: A review. J Anim Sci Biotechnol. 2015;6.

19. Farmer C. Nutritional impact on mammary development in pigs: a review. J Anim Sci. 2018;96:374856.

20. Rajasekar J, Perumal MK, Vallikannan B. A critical review on anti-angiogenic property of phytochemicals. J Nutr Biochem. 2019;71:1-15.

21. Tybirk PER. Nutrient Recommendations for pigs in Denmark. 17th ed. Copenhagen (Denmark): SEGES-VSP Danish Pig Research Centre; 2015.

22. FEDNA. Necesidades Nutricionales Para Ganado Porcino. 2nd ed. Madrid (Spain): Fundación Española para el Desarrollo de la Nutrición Animal. 2013. 
23. Krombeen SK, Bridges WC, Wilson ME, Wilmoth TA. Factors contributing to the variation in placental efficiency on days 70, 90, and 110 of gestation in gilts. J Anim Sci. 2019;97:359-73.

24. Karamysheva AF. Mechanisms of angiogenesis. Biochem. 2008;73:751-62.

25. Blavi L, Solà-Oriol D, Mallo JJ, Pérez JF. Anethol, cinnamaldehyde, and eugenol inclusion in feed affects postweaning performance and feeding behavior of piglets1. J Anim Sci. 2016;94:5262-71.

26. Fan Z, Xiao Y, Chen Y, Wu X, Zhang G, Wang Q, et al. Effects of catechins on litter size, reproductive performance and antioxidative status in gestating sows. Anim Nutr. 2015;1:271-5.

27. Meng Q, Guo T, Li G, Sun S, He S, Cheng B, et al. Dietary resveratrol improves antioxidant status of sows and piglets and regulates antioxidant gene expression in placenta by Keap1-Nrf2 pathway and Sirt1. J Anim Sci Biotechnol. 2018;9:34-47.

28. Kang K-S, Sun B, Aruoma OI, Fujii H, Bahorun T, Neergheen VS, et al. Low molecular proanthocyanidin dietary biofactor Oligonol: Its modulation of oxidative stress, bioefficacy, neuroprotection, food application and chemoprevention potentials. BioFactors. 2008;27:245-65.

29. Quesnel H, Farmer C. Review. Nutritional and endocrine control of colostrogenesis in swine. Animal. 2019;13:26-34.

30. Jamshidi N, Mantri N, Cohen MM. Acute effects of dietary plant nutrients on transcriptome profiles: evidence from human studies. Crit Rev Food Sci Nutr. 2020;60:1869-80.

31. Sirri R, Vitali M, Zambonelli P, Giannini G, Zappaterra M, Lo Fiego D, Pietro, et al. Effect of diets supplemented with linseed alone or combined with vitamin E and selenium or with plant extracts, on Longissimus thoracis transcriptome in growing-finishing Italian Large White pigs. J Anim Sci Biotechnol. 2018;9:1-13.

32. Reyer H, Zentek J, Männer K, Youssef IMI, Aumiller T, Weghuber J, et al. Possible Molecular Mechanisms by Which an Essential Oil Blend from Star Anise, Rosemary, Thyme, and Oregano and Saponins Increase the Performance and Ileal Protein Digestibility of Growing Broilers. J Agric Food Chem. 2017;65:6821-30.

33. Hansen SL, Trakooljul N, Liu H-C, Moeser AJ, Spears JW. Iron Transporters Are Differentially Regulated by Dietary Iron, and Modifications Are Associated with Changes in Manganese Metabolism in Young Pigs. J Nutr. 2009;139:1474-9.

34. Villodre Tudela C, Boudry C, Stumpff F, Aschenbach JR, Vahjen W, Zentek J, et al. Down-regulation of monocarboxylate transporter 1 (MCT1) gene expression in the colon of piglets is linked to bacterial protein fermentation and pro-inflammatory cytokine-mediated signalling. $\mathrm{Br} J$ Nutr. 2015;113:610-7.

35. Sargeant HR, Miller HM, Shaw MA. Inflammatory response of porcine epithelial IPEC J2 cells to enterotoxigenic E. coli infection is modulated by zinc supplementation. Mol Immunol. 2011;48:2113-21.

36. Lackeyram D, Yang C, Archbold T, Swanson KC, Fan MZ. Early Weaning Reduces Small Intestinal Alkaline Phosphatase Expression in Pigs. J Nutr. 2010;140:461-8.

37. Chmielewska M, Łosiewicz K, Podlasz P, Wasowicz K. The expression of mitochondrial, cytoplasmic and extracellular superoxide dismutase in the colonic wall of pigs suffering from swine dysenteria. 
Pol J Vet Sci. 2013;16:463-7.

38. Cockfield SM, Ramassar V, Noujaim J, Van der Meide PH, Halloran PF. Regulation of IFN-y expression in vivo: IFN-y up-regulates expression of its mRNA in normal and lipopolysaccharide-stimulated mice. J Immunol. 1993;150:717-25.

39. Pluske JR, Turpin DL, Kim JC. Gastrointestinal tract (gut) health in the young pig. Anim Nutr. 2018;4:187-96.

40. Heo JM, Opapeju FO, Pluske JR, Kim JC, Hampson DJ, Nyachoti CM. Gastrointestinal health and function in weaned pigs: a review of feeding strategies to control post-weaning diarrhoea without using in-feed antimicrobial compounds. J Anim Physiol Anim Nutr. 2013;97:207-37.

41. Spahn JM, Callahan EH, Spill MK, Wong YP, Benjamin-Neelon SE, Birch L, et al. Influence of maternal diet on flavor transfer to amniotic fluid and breast milk and children's responses: A systematic review. Am J Clin Nutr. 2019;109:1003S-1026S.

42. Kirsch F, Buettner A. Characterisation of the metabolites of 1,8-cineole transferred into human milk: Concentrations and ratio of enantiomers. Metabolites. 2013;3:47-71.

43. Song BJ, Jouni ZE, Ferruzzi MG. Assessment of phytochemical content in human milk during different stages of lactation. Nutrition. 2013;29:195-202.

44. Roura E, Koopmans SJ, Lallès JP, Le Huerou-Luron I, De Jager N, Schuurman T, et al. Critical review evaluating the pig as a model for human nutritional physiology. Nutr Res Rev. 2016;29:60-90.

45. Long G, Ao J, Wei H, Peng J, Tan C, Jiang S, et al. Effects of Dietary Supplementation of Oregano Essential Oil to Sows on Oxidative Stress Status, Lactation Feed Intake of Sows, and Piglet Performance. Biomed Res Int. 2015;2015:1-9.

46. Wang GY, Yang C, Yang Z, Yang W, Jiang S, Zhang G, et al. Effects of dietary star anise (Illicium verum Hook f) supplementation during gestation and lactation on the performance of lactating multiparous sows and nursing piglets. Anim Sci J. 2015;86:401-7.

47. Kim SW, Weaver AC, Shen YB, Zhao Y. Improving efficiency of sow productivity: Nutrition and health. J Anim Sci Biotechnol. 2013;4:2-9.

48. Berchieri-Ronchi CB, Kim SW, Zhao Y, Correa CR, Yeum K-J, Ferreira ALA. Oxidative stress status of highly prolific sows during gestation and lactation. Animal. 2011;5.

49. Wang X, Wu W, Lin G, Li D, Wu G, Wang J. Temporal proteomic analysis reveals continuous impairment of intestinal development in neonatal piglets with intrauterine growth restriction. J Proteome Res. 2010;9:924-35.

50. Gokce G, Haznedaroglu MZ. Evaluation of antidiabetic, antioxidant and vasoprotective effects of Posidonia oceanica extract. J Ethnopharmacol. 2008;115:122-30.

51. Murali R, Karthikeyan A, Saravanan R. Protective Effects of d-Limonene on Lipid Peroxidation and Antioxidant Enzymes in Streptozotocin-Induced Diabetic Rats. Basic Clin Pharmacol Toxicol. 2013;112:175-81. 
52. De Oliveira TM, De Carvalho RBF, Da Costa IHF, De Oliveira GAL, De Souza AA, De Lima SG, et al. Evaluation of p-cymene, a natural antioxidant. Pharm Biol. 2015;53:423-8.

53. Bischoff SC, Barbara G, Buurman W, Ockhuizen T, Schulzke JD, Serino M, et al. Intestinal permeability - a new target for disease prevention and therapy. Gastroenterol. 2014;14:1-25.

54. Song M, Lee JJ, Pettigrew JE, Maddox CW, Che TM, Liu Y, et al. Dietary plant extracts modulate gene expression profiles in ileal mucosa of weaned pigs after an Escherichia coli infection1. J Anim Sci. 2014;92:2050-62.

55. Markov AG, Aschenbach JR, Amasheh S. Claudin clusters as determinants of epithelial barrier function. Life. 2015;67:29-35.

\section{Tables}


Table 1. Ingredients and calculated nutrient compositions of gestation and lactation diets (as-fed basis)
Item
Gestation
Lactation

Ingredient composition, \%

\begin{tabular}{|c|c|c|}
\hline Barley & 35.00 & 10.00 \\
\hline Maize & 22.70 & 27.01 \\
\hline Wheat middling's & 15.00 & 7.00 \\
\hline Wheat & 9.00 & 25.55 \\
\hline Sunflower meal & 5.65 & 4.50 \\
\hline Sugar beet pulp & 3.10 & 2.50 \\
\hline Soybean meal, 47 \% CP & 2.50 & 13.50 \\
\hline Rapeseed meal & 2.50 & 4.50 \\
\hline Calcium carbonate & 0.99 & 1.25 \\
\hline Lard & 1.05 & 1.00 \\
\hline Dicalcium phosphate & 0.99 & 1.25 \\
\hline Salt & 0.40 & 0.50 \\
\hline L-Lysine $\mathrm{HCl}$ & 0.31 & 0.63 \\
\hline L-Threonine & 0.10 & 0.18 \\
\hline Mycofix plus 3. E & 0.10 & 0.10 \\
\hline Vit-Min Premix ${ }^{1}$ & 0.50 & 0.50 \\
\hline \multicolumn{3}{|l|}{ Calculated nutrient content } \\
\hline Net energy, kcal/kg & 2261 & 2455 \\
\hline Crude protein, \% & 13.0 & 16.7 \\
\hline Calcium, \% & 0.85 & 0.91 \\
\hline Total phosphorus, \% & 0.56 & 0.57 \\
\hline Dig. Phosphorus, \% & 0.35 & 0.37 \\
\hline SID Lysine, \% & 0.60 & 1.00 \\
\hline
\end{tabular}

${ }^{1}$ Supplied the following per kg of diet: vitamin A (retinyl acetate), $10000 \mathrm{IU}$; vitamin D3 (cholecalciferol), 2000 lU; vitamin E (acetate de tot-rac-3-tocopheryl), 45 mg; vitamin K3 (Menadione nicotinamide bisulphite), 3 mg; vitamin B1 (thiamine mononitrate), 3 mg; vitamin B2 (riboflavin), 9 mg; vitamin B6 (pyridoxine hydrochloride), 4.5 mg; vitamin B12 (cyanocobalamin), 0.04 mg; nicotinamide, 
$51 \mathrm{mg}$; pantothenic acid (calcium D-pantothenate), $16.5 \mathrm{mg}$; Biotin (D-(+)-biotin), $0.15 \mathrm{mg}$; folic acid, $1.8 \mathrm{mg}$; choline chloride, $350 \mathrm{mg}$; iron (as iron sulphate monohydrate), $54 \mathrm{mg}$; zinc (as zinc oxide), 66 $\mathrm{mg}$; manganese (as manganese oxide), $90 \mathrm{mg}$; iodine (as calcium iodine anhydrous), $1.2 \mathrm{mg}$; selenium (as sodium selenate), $0.18 \mathrm{mg}$; copper (as copper sulphate pentahydrate), $12 \mathrm{mg}$;

ethoxyquin, $4 \mathrm{mg}$; D,L-malic acid, 60 mg; fumaric acid, 75 mg; sepiolite, 907 mg; vermiculite 2001 mg; colloidal silica $45 \mathrm{mg} .63 \mathrm{ppm}$; citric acid, 8 ppm. 
Table 2. Effects of dietary BPC supplementation during gestation and lactation on performance of hyperprolific sows

\section{Treatment}

\begin{tabular}{|c|c|c|c|c|}
\hline Item & Control & BPC & SEM & $P$-value \\
\hline No. of sows & 20 & 20 & - & - \\
\hline Parity & 2.79 & 2.41 & - & - \\
\hline \multicolumn{5}{|l|}{ Sow BW, kg } \\
\hline Breeding (d 0) & 217.7 & 214.1 & 5.96 & 0.678 \\
\hline Farrowing standardized ${ }^{1}$ & 244.2 & 240.0 & 5.04 & 0.568 \\
\hline Weaning & 237.9 & 236.3 & 5.78 & 0.847 \\
\hline \multicolumn{5}{|l|}{ Sow back fat thickness, mm } \\
\hline Breeding & 14.17 & 13.73 & 0.736 & 0.674 \\
\hline Late gestation (d 110) & 14.04 & 13.58 & 0.731 & 0.662 \\
\hline Weaning & 12.58 & 12.52 & 0.588 & 0.949 \\
\hline \multicolumn{5}{|l|}{ Feed intake, kg } \\
\hline Gestation ADFI & 2.64 & 2.66 & 0.011 & 0.170 \\
\hline Lactation standardized ADFI (d 20) ${ }^{2}$ & $5.95^{y}$ & $7.34^{x}$ & 0.528 & 0.085 \\
\hline Standardized total feed intake ${ }^{3}$ & $425.2^{y}$ & $460.0^{x}$ & 12.51 & 0.071 \\
\hline Weaning to oestrus interval, $d$ & 3.79 & 4.05 & 0.267 & 0.845 \\
\hline
\end{tabular}

${ }^{1}$ Sow BW at gestation day 110 less litter birth weight.

${ }^{2}$ Lactation ADFI at day 20.

${ }^{3}$ Standardized total feed intake considered both the gestation and lactation at day 20 feed intake.

${ }^{x}, y_{M}$ eans within a row with different superscripts indicate significant trends $(P<0.10)$. 
Table 3. Effects of BPC supplementation during gestation and lactation on reproductive hyperprolific sows and litter performances

\begin{tabular}{|c|c|c|c|c|}
\hline \multirow[b]{2}{*}{ Item } & \multicolumn{4}{|c|}{ Treatment } \\
\hline & Control & BPC & SEM & $P$-value \\
\hline No. of sows & 14 & 14 & - & - \\
\hline Parity & 3.43 & 3.29 & - & - \\
\hline \multicolumn{5}{|l|}{ Sow reproductive performance } \\
\hline Litter birth weight, $\mathrm{kg}$ & 22.44 & 22.25 & 1.391 & 0.924 \\
\hline Total born piglets, $\mathrm{n}$ & $17.81^{y}$ & $19.96^{x}$ & 0.783 & 0.084 \\
\hline Piglets born alive, $\mathrm{n}$ & $14.69^{y}$ & $17.41^{x}$ & 0.825 & 0.077 \\
\hline Born alive piglet weight, kg & $1.33^{x}$ & $1.17^{y}$ & 0.068 & 0.092 \\
\hline \multicolumn{5}{|l|}{ Litter performance } \\
\hline Litter size at cross-fostering ${ }^{1}, \mathrm{n}$ & 14.54 & 14.73 & 0.173 & 0.448 \\
\hline Litter size at weaning, $\mathrm{n}$ & 14.34 & 14.50 & 0.242 & 0.651 \\
\hline Piglet cross-fostering weight, kg & 1.44 & 1.34 & 0.061 & 0.294 \\
\hline Piglet standardized weight $\mathrm{d} 20, \mathrm{~kg}$ & 4.80 & 5.11 & 0.230 & 0.370 \\
\hline Piglet weaning weight, $\mathrm{kg}$ & 5.27 & 5.62 & 0.183 & 0.197 \\
\hline Piglet weight gain from birth to weaning, $\mathrm{kg}$ & $3.90^{\mathrm{b}}$ & $4.47^{a}$ & 0.154 & 0.018 \\
\hline Lactation length, $d$ & 23.43 & 24.21 & 0.687 & 0.454 \\
\hline
\end{tabular}

a,b Means within a row with different superscripts indicate significant differences $(P<0.05)$.

${ }^{x, y}$ Means within a row with different superscripts indicate significant trends $(P<0.10)$.

${ }^{1}$ Litter sizes were adjusted by cross-fostering within treatment between days 1 and 2 after farrowing. 
Table 4. Effects of dietary BPC supplementation during gestation on jejunum histomorphology of neonate piglets

Treatment

\begin{tabular}{lllll}
\hline Item & Control & BPC & SEM & $P$-value \\
\hline Villus height, $\mu \mathrm{m}$ & $621^{\mathrm{b}}$ & $796^{\mathrm{a}}$ & 41.8 & $\mathbf{0 . 0 1 3}$ \\
\hline Crypt depth, $\mu \mathrm{m}$ & 65.3 & 67.8 & 1.50 & 0.279 \\
\hline Ratio V:C, $\mu \mathrm{m} / \mu \mathrm{m}$ & $9.8^{\mathrm{b}}$ & $12.3^{\mathrm{a}}$ & 0.66 & $\mathbf{0 . 0 2 4}$ \\
\hline $\begin{array}{l}\text { Goblet cell density/100 } \mu \mathrm{m} \\
\text { of villus height }\end{array}$ & $0.75^{\mathrm{b}}$ & $1.04^{\mathrm{a}}$ & 0.08 & $\mathbf{0 . 0 3 3}$ \\
\hline $\begin{array}{l}\text { Lymphocyte density/100 } \mu \mathrm{m} \\
\text { of villus height }\end{array}$ & 0.93 & & & \\
\hline
\end{tabular}

${ }^{a, b}$ Means within a row with different superscripts indicate significant differences $(P<0.05)$.

\section{Figures}

\section{$\square$ Placental fluid \&ilk}
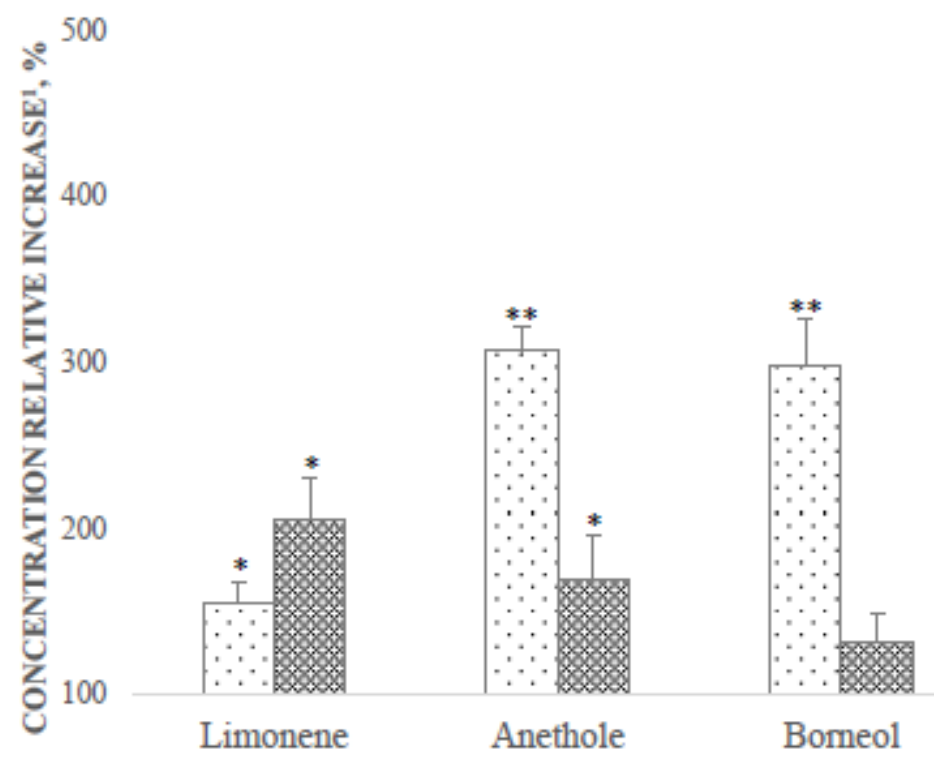

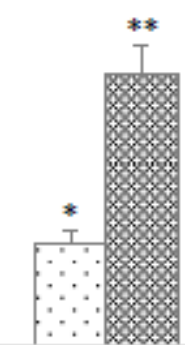

Bomeol

p-Cymene PHYTOGENIC COMPOUND

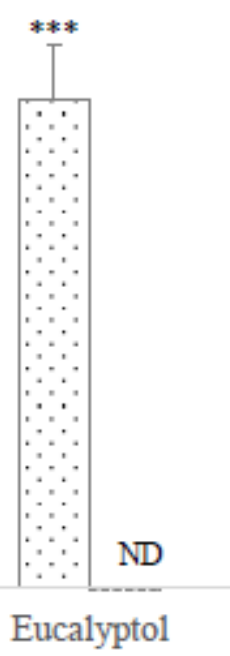

Eucalyptol

\section{Figure 1}

Relative concentration of BPC in placental fluid and milk of gestating and lactating hyperprolific sows. Values are expressed as percentage of concentration $\pm S E M, n=12$. ${ }^{\star} P<0.05 ; \star \star P<0.01 ; * \star \star P<.0001$. 
1Percentage of concentrations (abundance/retention time) relative increase respect to control (100\%). $\mathrm{ND}=$ not detected.

aControl $\square$ BPC

25

Colostrum

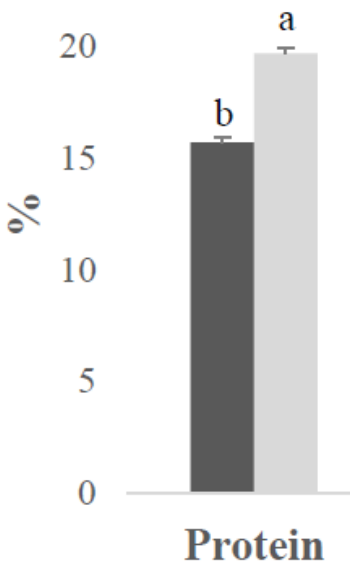

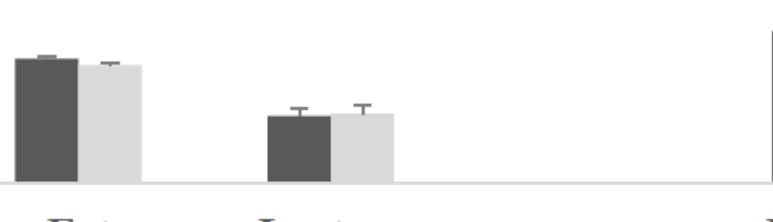

Fat

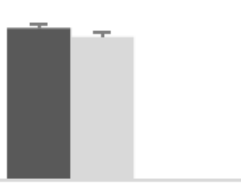

Protein
Milk

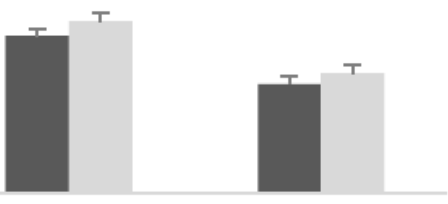

Fat Lactose

Figure 2

Effects of BPC supplementation during gestation and lactation on sow colostrum and milk composition. Values are expressed as LS means \pm SEM. a,bMeans with different superscripts indicate significant differences $(P<0.05)$. 
Sow antioxidant status
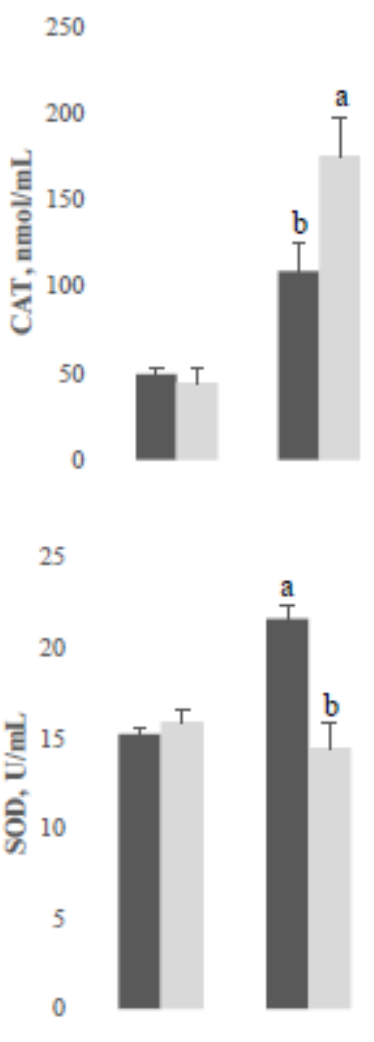

0,5

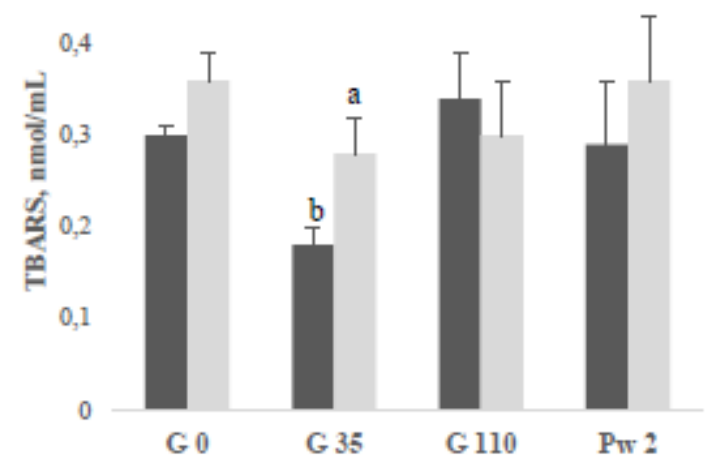

Piglet antioxidant status

250

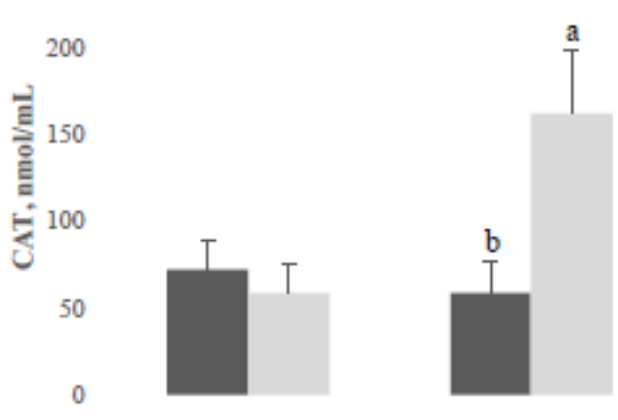

25

20
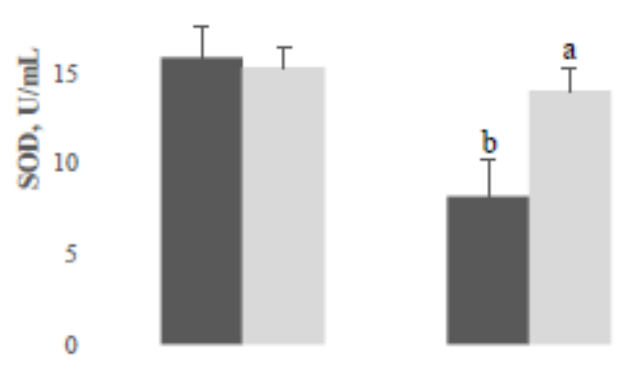

0,5

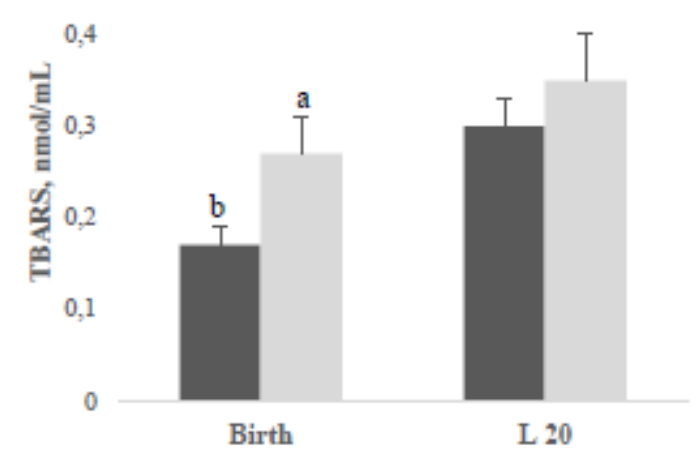

\section{Figure 3}

Antioxidant status of sows and piglets by BPC supplementation during gestation and lactation against unsupplemented. Control, not supplemented; BPC, PC supplemented during gestation and lactation. Sow antioxidant status: $\mathrm{G} 0$, gestation day $0(n=20) ; \mathrm{G} 35$ = gestation day $35(n=15) ; \mathrm{G} 110$, gestation day $110(n=14)$; Post-w d2, post-weaning day $2(n=14)$. Piglet antioxidant status: Birth, neonate piglets without suckling $(n=8) ; L 20$, lactation day $20(n=8)$. Values are expressed as LS means SEM. 2-Means with different superscripts indicate significant differences $(P<0.05)$. 


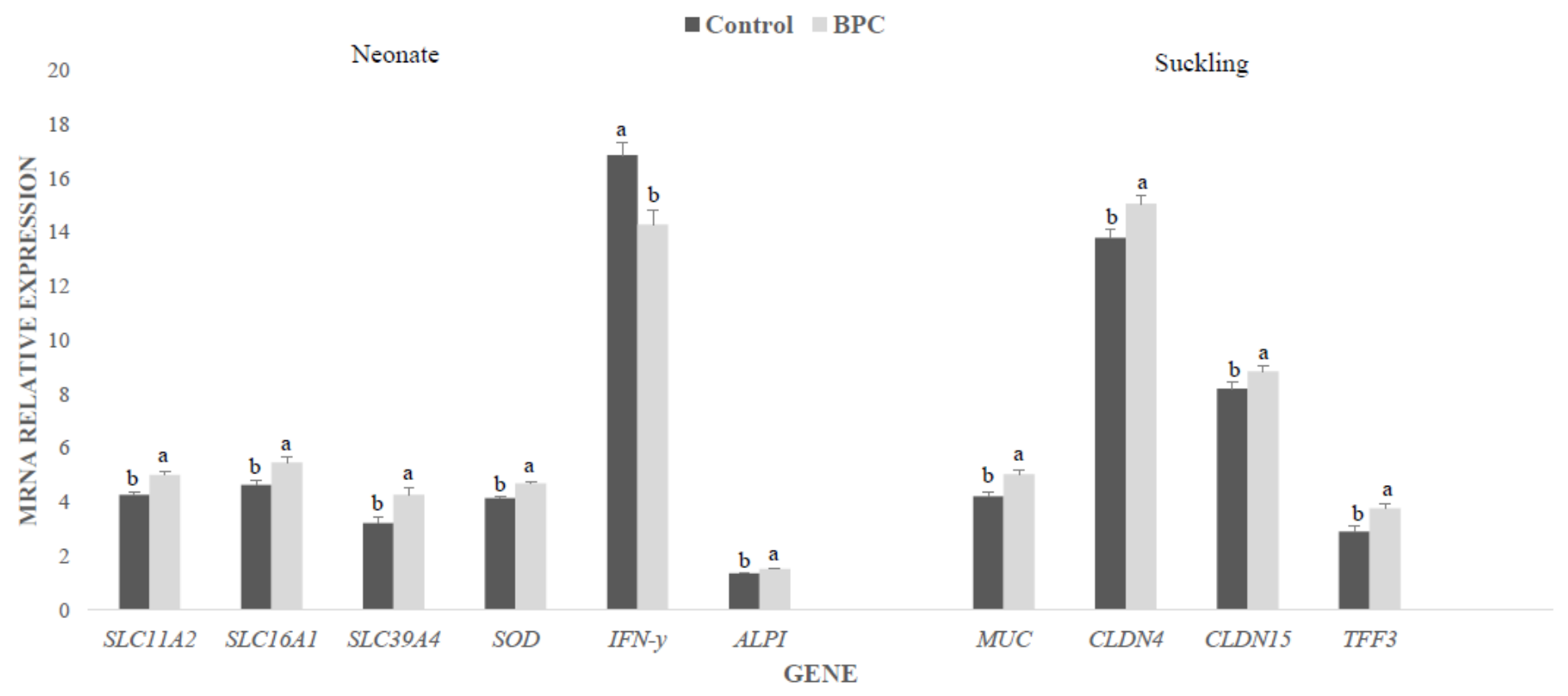

\section{Figure 4}

Effects of sow's dietary BPC supplementation on jejunum mRNA expression in neonate, and suckling piglets. Neonate piglet: Nutrient transporter genes: SLC11A2, solute carrier family 11 (proton-coupled divalent metal ion transporter) member 2; SLC16A1, monocarboxylate transporter 1; SLC39A4, solute carrier family 39 (zinc transporter) member 4. Antioxidant gene: SOD, superoxide dismutase. Immune response gene: IFN- $y$, interferon gamma. Digestive gene: ALPI, intestinal alkaline phosphatase. Suckling piglet: Barrier function genes: MUC, mucin 2; CLDN4,

\section{Supplementary Files}

This is a list of supplementary files associated with this preprint. Click to download.

- SupplementaryTable1.docx 